\title{
Desafiando la formación de psicólogos organizacionales desde una perspectiva de aprendizajes situados en el ámbito micro-empresarial peruano*
}

\author{
Defying the training of organizational psychologists from a situated learning perspective in the Peruvian micro- \\ enterprise environment
}

Desafiando a formação de psicólogos organizacionais desde uma perspectiva de aprendizagens situadas no entorno microempresarial peruano

Ángela Vera Ruiz ${ }^{\mathrm{a}}$

Pontificia Universidad Católica del Perú, Perú

averar@pucp.pe

ORCID: http://orcid.org/0000-0002-8491-3979

César Andrés Llanco Gonzales

Pontificia Universidad Católica del Perú, Perú

ORCID: http://orcid.org/0000-0001-8490-7096

Luis Alberto Guevara Rojas

Pontificia Universidad Católica del Perú, Perú

ORCID: http://orcid.org/0000-0001-5520-7402
DOI: https://doi.org/10.11144/Javeriana.cao32-58.dfpo Redalyc: http://www.redalyc.org/articulo.oa?id=20560207008

Fecha de recepción: 30/06/2018

Fecha de aprobación: 29/03/2019

Fecha de publicación: 30/06/2019

\section{Resumen:}

Este artículo presenta una experiencia de formación académica, donde estudiantes de pregrado de psicología organizacional, de una universidad peruana, hacen su práctica profesional en microempresas para analizar el comportamiento organizacional. Bajo un enfoque de responsabilidad social universitaria se reconoce a las microempresas como actores importantes en la economía nacional. Con la vinculación entre estudiantes y microempresas se busca decolonizar conocimientos hegemónicos, impartidos en la formación tradicional, evidenciando la necesidad de pensar los saberes y prácticas organizacionales de una manera situada, crítica y compartida por todos los participantes. Se plantean los principales aspectos metodológicos y resultados generales operativos de la experiencia, como insumo pedagógico útil en contextos formativos del complejo e inequitativo mundo laboral y organizacional latinoamericano.

Códigos JEL: D21, D23, M19

Palabras clave: decolonización, formación en psicología organizacional, microempresas.

\section{Abstract:}

This paper presents a formative academic experience where undergraduate students of organizational psychology from a Peruvian university do their internship in local microenterprises. They conducted participant observation in order to analyze microenterprises' organizational behavior. By using a university social responsibility approach, the project recognizes microenterprises as the prevailing type of organization in the national economy. Through the linkage of students to the microenterprises, this project deconstructs and decolonizes the hegemonic knowledge traditionally taught in business schools, demonstrating the need to think about organizational knowledge and practices in a way that is situated, critical and shared by all participants. Methodological details and general operative results of this experience are outlined, as a useful pedagogical input in training contexts of the complex and inequitable labor and organizational Latin American framework.

JEL Codes: D21, D23, M19

Keywords: decolonization, training in organizational psychology, microenterprises, situated learning.

\section{Resumo:}

Este artigo apresenta uma experiência de formação acadêmica, na qual estudantes de graduação em psicologia organizacional de uma universidade peruana fazem sua prática professional em microempresas para analisar o comportamento organizacional. Sob

Notas de autor:

a Autora de correspondencia. Correo electrónico:averar@pucp.pe 
um enfoque de responsabilidade social universitária se reconhece às microempresas como atores importantes na economia nacional. Com a vinculação entre estudantes e microempresas se procura descolonizar conhecimentos hegemônicos, impostos na formação tradicional. Se evidência, assim, a necessidade de pensar os saberes e práticas organizacionais de uma maneira situada, crítica e compartilhada por todos os participantes. Expõem-se os principais aspectos metodológicos e os resultados generais operativos da experiência. Estes servem como insumo pedagógico útil em contextos formativos do complexo e desigual mundo laboral e organizacional latino-americano.

Códigos JEL: D21, D23, M19

Palavras-chave: descolonização, formação em psicologia organizacional, microempresas.

\section{Introducción}

Cuando las universidades y los centros de formación superior latinoamericanos no hacen una reflexión crítica y aguda a sus prácticas docentes, pueden convertirse en legitimadores de saberes considerados neutrales y universales, producto de la colonización del conocimiento hegemónico, olvidando los contextos en que se imparten y en los cuales se desempeñarán los profesionales (Castro-Gómez, 2007; Lander, 2000; Mandiola, 2018). Se disemina, entonces una racionalidad específica, originada en los países del norte, que avasalla a otro tipo de saberes locales, ignorando su voz y sustento en las necesidades de las personas que los articulan (Quijano, 2000a).

Para el caso particular de la formación en el campo de la administración y las prácticas organizacionales, el riesgo de una formación superior acrítica se exacerba cuando se asumen como objetivos y universales los desarrollos conceptuales y prácticos sobre las dinámicas de gestión organizacional, producidos en contextos nor-atlánticos, donde las problemáticas sociales, económicas y laborales son distintas a las de la realidad laboral y organizacional latinoamericana (Mandiola, 2013, 2018; Pulido-Martinez \& Sato, 2013; PulidoMartinez, 2004).

En este documento se presenta una experiencia de formación académica, realizada en una universidad peruana, en el marco de un curso de formación de pregrado para futuros psicólogos sociales que, bajo el enfoque de Responsabilidad Social Universitaria - RSU- ha buscado incorporar en sus prácticas formativas elementos de aprendizaje sobre las problemáticas sociales locales, buscando promover un posicionamiento crítico en los estudiantes, respecto al rol de la psicología en los ámbitos laborales (Pulido-Martinez, 2004).

Dicha experiencia académica parte del reconocimiento de las características de las organizaciones locales, en su mayoría microempresas (Alva, 2017; Arbulú \& Otoya, 2006; Campaña, 2013) y promueve la aproximación a un conocimiento que se produce en el trabajo conjunto y horizontal entre microempresarios y estudiantes del curso, para generar aprendizaje críticos, reflexivos y socialmente relevantes, que se pongan al margen del conocimiento tradicional del management (Mandiola, 2018). Precisando así saberes integrados a las prácticas y necesidades organizacionales locales, decolonizando los conceptos tradicionalmente transmitidos y consumidos de forma acrítica sobre los alcances de la psicología del trabajo y las organizaciones (Pulido-Martinez \& Sato, 2013; Stecher, 2013).

En el Perú se viven, de manera cotidiana y naturalizada, diferentes tipos de exclusión asociados a raza, género, nivel de estudios, nivel de ingresos y condiciones laborales, que perpetúan asimetrías y brechas en la sociedad (Arellano, 2010; Bruce, 2007; Espinosa, Calderón, Burga \& Güímac, 2007; Espinosa \& Cueto, 2014). El uso de estereotipos y conductas discriminadoras afectan el desarrollo laboral de personas y poblaciones estigmatizadas, poco visibilizadas como sujetos de interés para el marco institucional, privado y estatal, que por ende aparecen marginalmente en la agenda de investigación de los procesos organizacionales en el país (Kogan, Fuchs \& Lay, 2013). Esto tiene como consecuencia bajos niveles de inclusión, productividad, bienestar y acceso a oportunidades laborales para un alto porcentaje de la población que busca empleo (Amat y León, 2012; Campaña, 2013). 
Los grupos poblacionales no incluidos en el sistema laboral tradicional han introducido su fuerza productiva apelando a estrategias de autoempleo, en algunos casos desde la informalidad, viendo su mayor cristalización en iniciativas microemprendedoras, desde las cuales se satisfacen las demandas de inclusión económica en una sociedad altamente desigual y cambiante (Amorós \& Bosma, 2013; Arellano, 2010; Campaña, 2013; INEI, 2018. Estos sectores laborales, menos visibilizados y atendidos en el marco de las temáticas de los estudios del management tradicional y de la psicología del trabajo y las organizaciones, son paradójicamente los que reclutan mayor densidad de población económicamente activa y mantienen la mayor tasa de empleos en el país. Más del $70 \%$ de la población trabajadora peruana general y, específicamente, el $88,2 \%$ de la población pobre del país labora en una microempresa (Alva, 2017; Comex, 2017; INEI, 2018.

En el Perú las microempresas son identificadas como unidades de producción económica que cuentan con menos de 10 empleados y generan ventas anuales menores a S/525,000 (aproximadamente 175,000 dólares (Arteaga, 2017. Aunque estas constituyen alrededor del 97\% de las empresas del país y existe una ley que busca "la promoción de la competitividad, formalización y desarrollo de las micro y pequeñas empresas para incrementar el empleo sostenible, su productividad y rentabilidad” (Ley 2828015 de 2003, su aporte al PIB es sólo del 30\%, porque siguen teniendo poco acceso a sistemas de financiamiento, capacitación y formalización, por lo que sus niveles de desarrollo y acceso a oportunidades son restringidos, revirtiendo, también, en menores posibilidades de incrementar los ingresos y calidad de vida de sus colaboradores (Alva, 2017; Hernani \& Hamann, 2013; INEI, 2018; Ravelo, 2012

Diferentes estudios han identificado características comunes en los procesos internos de las microempresas, entre ellas: no separar las finanzas domésticas de las del negocio, predominancia femenina (entre un 30 a 60\%; dependencia de la mano de obra familiar (Ameconi, 2004 y precariedad de recursos materiales y productivos dada la baja inversión, producto del restringido acceso a préstamos fi nancieros porque el sistema bancario formal no los admite como sujetos económicos (Zamora, 2017. Y aunque su relevancia para la economía nacional es indiscutible, no sólo por su contribución a la generación de empleo, sino también por su participación en el desarrollo socioeconómico de las regiones en donde se ubican (Avolio, Mesones \& Roca, 2011; Tello, 2014 las microempresas enfrentan obstáculos tales como: informalidad, desarticulación empresarial, uso inadecuado de la tecnología, dificultad de acceso a fi nanciamiento, escasas capacidades operativas, limitadas capacidades gerenciales y problemas de acceso a la información (Matute, Bohorquez, Carbajal, Díaz, Espinoza, \& Jiménez, 2008.

El reconocimiento de este contexto de empleabilidad concentrada en las microempresas, sumado a la necesidad de reflexionar sobre un país con altos índices de exclusión y discriminación, debería ser fundamental para el desarrollo de la investigación en management local y, ante todo, debería ser parte de la formación consciente y situada de los profesionales que se van a desempeñar laboralmente en Perú. Es así que este estudio rescata el importante lugar que juegan las microempresas en términos de empleabilidad y productividad económica de un amplio sector de la población peruana y, en especial, de la población en condición de pobreza (INEI, 2018 Y desde un enfoque de RSU los microempresarios son considerados actores con potencial para favorecer el cambio y la inclusión social desde sus iniciativas empresariales, muchas veces no visibilizadas en las temáticas de investigación más amplias y tradicionales del campo organizacional (Hernani \& Hamann, 2013; Portocarrero, Tarazona \& Camacho, 2006

Este proyecto pretende dar voz a aquellos actores invisibilizados desde el conocimiento hegemónico racional tradicional (Quijano, 2000b, para reconocer a los verdaderos protagonistas de la escena empresarial y empleadora peruana: los microempresarios. Se busca que estos actores mantengan comunicación y colaboración permanente con los estudiantes del curso para construir, desde sus saberes, una nueva manera de entender el quehacer de las organizaciones locales y la pertinencia de la psicología social del trabajo en la identificación y atención de las necesidades de sus empresas. Lo que implica reevaluar las teorías clásicas del campo del comportamiento organizacional con las que tradicionalmente se trabaja, para promover conocimientos que emerjan en el diálogo directo, profundo y respetuoso con los espacios microempresariales. 


\section{El desafío a la formación en psicología organizacional local}

La propuesta académica-formativa que se ofrece en el campo de la psicología de las organizaciones y del trabajo en el Perú, se orienta predominantemente a la administración y evaluación del desempeño efectivo de los trabajadores; por lo que promueve la apropiación de técnicas de evaluación y categorización de las personas, a través de procesos validados, como el diseño de vacantes laborales, reclutamiento, selección de personal y evaluación del desempeño (Elizade, Mollo \& Flores, 2010; León, 2013).

Esta alineación de la formación en psicología al campo administrativo tradicional, conlleva una dificultad para interpelar a los profesionales de la psicología organizacional a buscar un equilibrio para atender los intereses productivos de la organización, sin olvidar que las personas que la componen también tienen necesidades de bienestar y no son meros recursos humanos (Highhouse \& Schmitt, 2013). La interpelación a fomentar prácticas organizacionales más humanas y críticas exige que la formación universitaria en psicología organizacional brinde una visión de carácter crítico y socialmente responsable, para que los psicólogos integren dos roles o escenarios, aparentemente separados, que suelen confrontar la integridad de su ejercicio profesional: por un lado, el rol científico enfocado en el desarrollo de teorías que básicamente se queda en los alcances del ámbito académico; por otro lado, se reconoce el rol profesional práctico, dedicado a la solución de problemas de la vida real organizacional que, en muchos casos, opera desde el sentido común (Carvajal, Ulloa, \& Morales, 2007; Pulido-Martínez, 2004; Pulido-Martinez \& Jaraba, 2017).

El desafío del proyecto académico, cuyos aspectos prácticos/metodológicos se presentarán en este documento, es justamente cuestionar, de manera crítica, las estrategias de educación que reciben los jóvenes universitarios, formándose como futuros profesionales que ejercerán la psicología en el campo del trabajo y las organizaciones. En ese sentido, el increpar los formatos estándares educativo-formativos, lleva necesariamente a apelar a la desnaturalización del conocimiento que tradicionalmente se imparte en las aulas universitarias, donde los jóvenes profesionales justificarán un futuro ejercicio laboral de acuerdo con los marcos conceptuales y metodológicos -generalmente hegemónicos y descontextualizados de la realidad local- que les brinda la academia (Castro-Gómez, 2007; Lander, 2000).

El proyecto analiza la configuración de la identidad posibilitada en y para el contexto productivo; reconociendo que el conocimiento psicológico, capaz de problematizar su "domesticación”, de manera crítica, tiene mucho que aportar a la comprensión de los procesos individuales y sociales que se gestan en los entornos laborales y productivos (Pulido-Martinez \& Sato, 2013); movilizando escenarios laborales más conscientes y justos que reviertan en beneficios sociales para la comunidad (Veronese \& Guareschi, 2005).

Nuestra propuesta académica reconoce la urgencia de formar profesionales en psicología organizacional conscientes, críticos y socialmente responsables, capaces de analizar de manera compleja cuáles son las dinámicas en las que se hacen posibles distintas formas de organizarse para trabajar y ser productivos en el marco económico imperante (Stecher, 2013; Miller \& Rose, 2009; Gergen, 2007). El proyecto considera, ante todo, la peculiaridad de las organizaciones con las que se trabaja, las cuales, dado su tamaño micro y sus relaciones un tanto informales, configuran modos alternos de comportamiento organizacional a los referidos en la teoría hegemónica. Por lo que el trabajo con este tipo de contexto laboral pone en evidencia algunas limitaciones de los modelos conceptuales generados en contextos primermundistas, lo que enfatiza el carácter decolonial del conocimiento que se produce en este proyecto, donde los actores involucrados se ven en la necesidad de construir sus propias categorías y marcos comprensivos, al margen de la teoría tradicional, para poder entender, de manera orgánica (Castro-Gomez, 2007) procesos que no calzan en las dimensiones del comportamiento organizacional, convencionalmente explicadas en los libros de texto mainstream.

Con el proyecto se busca que los estudiantes desarrollen una perspectiva teórica, situada y coherente en la cual reflexionen sobre la pertinencia de los conocimientos y prácticas psicológicas para el contexto laboral de las microempresas peruanas, cuestionando el ejercicio del conocimiento psicológico en la perpetuación de prácticas de corte funcionalista y gerencial, centradas en el modelo de la productividad. El proyecto dialoga 
también con los estudios críticos sobre educación en el ámbito empresarial y de gestión, para proponer pedagogías que vinculen explícitamente los aspectos sociales, históricos y políticos asociados a las prácticas del magement (Mandiola, 2013.

\section{Aspectos metodológicos para facilitar aprendizajes compartidos y situados}

La propuesta académica, avalada por la Dirección de Responsabilidad Social de la Universidad, se ha ejecutado entre los años 2016 y 2018, en el marco del curso socialmente responsable "Comportamiento Organizacional” - $\mathrm{CO}$-, obligatorio en el último año de formación de la especialidad de psicología social, antes de iniciar las prácticas pre-profesionales. A continuación se describen los principales aspectos metodológicos considerados para la ejecución exitosa del proyecto, garantizando el cumplimiento de su principal objetivo de aprendizaje que es facilitar aprendizajes colaborativos, respetuosos y situados, que den lugar a la reflexión crítica sobre la contextualización y viabilidad de la aplicación de los conocimientos del $\mathrm{CO}$, en un trabajo participativo con microempresas de zonas aledañas al campus universitario.

Los objetivos específicos son: 1 Promover un aprendizaje reflexivo, colaborativo y consciente de la realidad organizacional local, fortaleciendo en los estudiantes habilidades investigativas que les permitan comprender cómo se configura el $\mathrm{CO}$ en microempresas locales, desde el uso de metodologías participativas. 2 Favorecer el comportamiento ético y de ciudadanía en los estudiantes que posibilite la consolidación de compromisos, brindando a los microempresarios un diagnóstico de calidad, generado en la comprensión de la realidad singular del comportamiento organizacional de cada empresa. 3 Fomentar experiencias de participación en proyectos de RSU en los estudiantes, con un fin pedagógico y formativo de compromiso social y de decolonización del conocimiento.

\section{Participantes}

El proyecto cuenta con cuatro versiones semestrales ejecutadas, cada una de ellas con su respectivo grupo de microempresas y estudiantes. En total han participado 91 estudiantes (aproximadamente 23 por semestre de noveno ciclo de la carrera de psicología social, quienes llevan el curso durante un semestre académico (16 semanas. Los estudiantes son informados con detalle sobre las condiciones, objetivos y metodología del curso y se organizan voluntariamente en equipos de tres a cinco participantes, para desarrollar el trabajo progresivo a lo largo del curso. Todos los estudiantes reciben capacitación previa a la salida de campo y son monitoreados y asesorados, en cada uno de los avances del proceso, por el equipo docente, constituido por un profesor de teoría y un asesor de práctica.

A cada equipo de trabajo de estudiantes se le asigna una microempresa, previamente identificada, contactada e informada sobre el proyecto, cuyo dueño/a -tras la firma de un consentimiento informado y una inducción al proyecto- acepta participar de manera voluntaria y colaborativa comprometiéndose a recibir al grupo de estudiantes en sus instalaciones, al menos una vez a la semana durante el semestre, garantizando su tiempo y el consentimiento de sus colaboradores, para brindar la información y el espacio donde los estudiantes puedan realizar el proceso. Como principio ético de beneficencia, el empresario recibe, al final del semestre, un informe-diagnóstico del CO, con sugerencias para mejorar su organización. Se ha trabajado con un total de 20 microempresas (cinco empresas por semestre en diferentes rubros, todas ellas ubicadas en zonas aledañas al campus de la Universidad.

Los roles de todos los involucrados en el proyecto: estudiantes, empresarios y docentes, son altamente participativos, horizontales, de mutuo aprendizaje y disposición a la construcción de saberes compartidos. La realización exitosa del curso conlleva un alto nivel de planificación y coordinación de voluntades, por lo que la comunicación es un aspecto clave para el logro de los objetivos. 


\section{Instrumentos}

Los estudiantes que se vinculan al curso se comprometen a realizar, durante un semestre académico, un trabajo de campo grupal, nutrido por una serie de guías de observación, guías de entrevistas e instrumentos de medición, asociados a los constructos teóricos centrales del CO tradicional (Robbins \& Judge, 2013). Las herramientas de recolección de información corresponden a los objetivos del curso, reconociendo la perspectiva de enfoque etnográfico como la metodología más pertinente y coherente con los alcances del curso y la experiencia reflexiva, critica, vivencial e interpretativa que se busca promover (Geertz, 1996; Guber, 2001).

Se han mejorado las herramientas utilizadas cada semestre y se ha validado el material para la realización óptima del trabajo de campo, además del diseño de guías semanales que orientan la labor de los estudiantes en las empresas y que les permite organizar la información recopilada en cada uno de los temas del Comportamiento Organizacional -CO- concentrándose en tres ejes principales (Robbins \& Judge, 2013):

1. El CO desde una perspectiva sistémica, epistemológica y metodológica, que implica una noción interpretativa de la Cultura Organizacional, transversal a todo el proyecto, en la que se fundamentan las guías de observación participante (Gutiérrez, 2007).

2. El CO desde una perspectiva individual que abarca el uso de instrumentos cuantitativos y cualitativos para evaluar rasgos de personalidad, valores, satisfacción laboral y comportamientos de ciudadanía organizacional en los miembros de la organización.

3. El CO desde una perspectiva grupal y de interacciones que trabaja actividades y entrevistas grupales para analizar temas de equipos, comunicación, liderazgo y poder.

En busca de potenciar la participación de los empresarios se ha diseñado un formato de supervisión de trabajo de campo y un cronograma de visitas que es diligenciado y firmado por ellos cada vez que los estudiantes visitan su organización. En dicho formato se monitorean los avances de los estudiantes en la empresa, comentando las condiciones en que se hizo la observación y las sugerencias de aspectos por mejorar.

También se cuenta con formatos de evaluación interna del proceso con los estudiantes, adicionales a las evaluaciones/rubricas del trabajo en el curso, que, de manera anónima recogen las impresiones, vivencias, objeciones y recomendaciones de los estudiantes al inicio, en la mitad y al final del proceso. Permitiendo ver los progresos en la forma de concebir y relacionarse con el trabajo, con los conocimientos y con las empresas.

\section{Procedimiento}

Como fundamento metodológico el proyecto apela a una comprensión interpretativa y densa de los procesos sociales e individuales que surgen en las organizaciones, por medio de un trabajo exhaustivo de campo y observación participante (Geertz, 1996; Guber, 2001, 2004), con el que se busca entender la manera en que se configuran realidades organizacionales, entendidas como culturas, en las que se consolidan identidades y subjetividades socialmente validadas para el entorno laboral inmediato (Gutiérrez, 2007). El curso se vale de una metodología práctica, participativa y analítica que implica la necesidad de salir del espacio del aula a la realidad local de las microempresas, para visualizar escenarios de aprendizaje aplicativo y diálogo constructivo, que favorezcan la comprensión de los fenómenos del CO desde las necesidades puntuales de contexto productivo local (ver el Anexo 1).

Los equipos de estudiantes realizan, como mínimo, una visita semanal a la microempresa con el fin de recabar información sobre objetivos y contenidos específicos que están siendo reflexionados en el espacio teórico del curso y que, si bien se plantean desde la teoría canónica del CO, resultan siendo rebatidos, problematizados y resignificados en el contraste con la experiencia vivida y recogida en el trabajo de campo. 
El curso cuenta con dos espacios pedagógicos, además del trabajo de campo: tres horas teóricas y dos horas de asesoría de práctica semanales. En el primer espacio se revisa la teoría hegemónica y, a la vez, se discuten estudios y análisis de corte crítico y reflexivo sobre el uso de la misma y las implicaciones que ésta tiene en los modos de concebir el ámbito organizacional. Este espacio también es utilizado para generar informes del trabajo de campo y socializar los hallazgos y reflexiones entre los estudiantes con el objetivo de compartir aprendizajes y orientar posibilidades de interpretación y reflexión de la información recogida más allá de los marcos teóricos desarrollados en el curso.

En el segundo espacio pedagógico, de asesoría de práctica, se acompaña de manera conjunta y personalizada a cada grupo de estudiantes según los avances que van sistematizando en un informe de investigación que, al finalizar el ciclo, constituye la herramienta de diagnóstico organizacional a entregar al empresario participante. Además de trabajar la aplicación práctica de los contenidos conceptuales del curso, se busca reforzar la capacitación de los estudiantes en técnicas de recolección (entrevista y observación y análisis de información con el fin de incrementar el impacto del trabajo de campo y del análisis de los resultados de la observación.

Por su parte, los empresarios participantes cumplen un papel activo y formador en el proceso de los estudiantes ya que, desde sus propias experiencias empíricas, enriquecen los marcos investigativos en los que se sitúan las guías y los constructos que orienta el curso, dando lugar a la reflexión conjunta que se logra en los procesos de validación y retroalimentación de la información y análisis que van llevando a cabo los estudiantes en su trabajo de campo. Aproximadamente cada tres semanas de campo, los estudiantes hacen un informe y presentan puntos de devolución al empresario para validar la información recogida y profundizar en la comprensión de los procesos que se van identificando en la organización.

Los empresarios y empresarias participantes poseen conocimientos prácticos muy valiosos, que no necesariamente son visibilizados en las teorías trabajadas en clase y que resultan claves para enriquecer la visión analítica del estudiante. Es por esto que, durante esta experiencia, sus saberes aportan en la generación de nuevo conocimiento de la realidad de las microempresas peruanas, contribuyendo a la comprensión de las empresas en el país.

Al final del semestre se lleva a cabo un evento de cierre a manera de conversatorio en el que participan los estudiantes, los empresarios y miembros de la comunidad académica y empresarial interesados en los temas, con miras a compartir los avances del proyecto y visualizar perspectivas de mejora, pertinencia y articulación del mismo con otros ámbitos de la Universidad y la comunidad. Vale señalar que el proceso de trabajo de campo es evaluado a lo largo del semestre tanto en el aprendizaje de los estudiantes como en el compromiso de los empresarios, con el fin de poder demostrar con evidencias que la participación en el curso reporta aprendizajes y beneficios para ambas partes. De esta manera, se busca hacer un trabajo ético, reflexivo y riguroso que se compromete con los principios de justicia, beneficencia y aporte social de la ética en la investigación.

\section{Decolonizar los espacios de formación organizacional}

Uno de los resultados más significativos del curso, afín con los objetivos, es que los estudiantes demuestran, a través de los avances del proyecto y la realización del diagnóstico final, que la dinámica colaborativa y de campo favorece aprendizajes reflexivos, con los que pueden cuestionar y/o enriquecer la teoría y constructos que suelen enseñarse como universales para las dinámicas organizacionales. Lo que supone generar interpretaciones y comprensiones situadas, para pensar otros modos de conceptualizar las prácticas que identifican en su trabajo de campo con las microempresas.

El curso cuenta con instrumentos que, por un lado, recopilan las preconcepciones de los estudiantes acerca del $\mathrm{CO}$ con las que entran al curso y, por otro, recogen los aprendizajes que obtienen durante el proyecto 
y testimonios sobre el cumplimiento de sus expectativas del curso. El curso exige el involucramiento de los docentes para guiar y retroalimentar el avance de cada grupo de estudiantes, reconociendo sus motivaciones, emociones, conteniéndoles y protegiendo su integridad ante las demandas del campo. Por tratarse de un proceso dinámico y cualitativo donde las circunstancias del campo pueden cambiar repentinamente (el cierre de la empresa, la cancelación imprevista de una visita por parte del empresario), se requiere promover en los estudiantes un nivel adecuado de proactividad y tolerancia a la frustración que les permitan extraer, de cada experiencia del curso, aprendizajes para su formación.

El factor de informar previamente a los estudiantes sobre las características del proyecto, las implicaciones a nivel de horas de trabajo, costos, beneficios y compromisos que ello conlleva, clarifica el ánimo y las expectativas que ellos tienen respecto al curso y potencia su agencia y apropiación activa del conocimiento a lo largo del semestre. En general, las experiencias han sido muy positivas y los reportes de los estudiantes evidencian una satisfacción alta con el reto que el curso les plantea y con la coherencia y rigurosidad que el mismo mantiene en toda su ejecución.

El trabajo de campo cumplió con todas las expectativas que tenía del curso. Me encantaron las actividades y todas las visitas que hicimos a la empresa. Siento que aprendí mucho porque todo el curso está realmente organizado y desde la primera práctica se nos dijo todo lo que tendríamos que hacer. Fue genial. (Estudiante, 2018-1)

La noción de psicología organizacional que tienen los estudiantes, al iniciar el curso, se reduce a la imagen de una empresa grande y estática donde el rol del psicólogo se limita a procesos de selección, clima, desempeño. Durante el curso, los estudiantes logran identificar nuevos elementos compresivos del ámbito organizacional (el elemento "cultura" es el que tiene mayor predominancia) y tener una visión dinámica del funcionamiento de la empresa y las organizaciones, reconociendo niveles de interacción, individual, grupal y estructural (Robbins \& Judge, 2013) donde la influencia y el aporte del trabajador es indispensable, por lo que no puede ser visto como un mero "recurso" pasivo (Gutiérrez, 2007). La experiencia de campo ha tenido como principal punto a favor su grado de novedad y cercanía con el contexto práctico.

Me encanta el proyecto, es una perspectiva distinta que te hace tener un panorama más grande sobre lo que es una organización y cómo la psicología entra a tallar allí en su responsabilidad con las empresas locales. (Estudiante 2017)

Creo que la parte práctica es lo mejor del curso porque podemos entender y aplicar cómo funcionan, o no, todos los conceptos y las teorías que nos enseñan en clase, y esto nos pone a pensar "fuera de la caja". (Estudiante, 2016)

Los estudiantes reconocen que se trata de una experiencia diferente cuyo contenido se va estructurando conforme se recolecta la información en las visitas y las entrevistas. Tras el análisis y sistematización de los resultados, ellos pueden reconocer las diferencias entre la conceptualización occidental del comportamiento organizacional frente a los hallazgos en las microempresas. Los estudiantes del curso entienden que los elementos identificados como base de determinados procesos desde la literatura mainstream, pueden manifestarse de manera diferente a lo esperado y mantener su funcionalidad dentro de la organización microempresarial. Elementos como el liderazgo, el trabajo de equipos o la satisfacción laboral, entre otros, exigen ser leídos de manera diferente, en un ámbito microempresarial con vínculos familiares y orgánicos diferentes. Este nivel de reflexión logrado por los estudiantes, define el rol decolonial del proyecto, pues se plantea un desafío a las teorías tradicionales al leerlas en contexto, poniendo en valor la necesidad de generar conocimientos, con sus consecuentes prácticas y adecuada a la realidad local (Mandiola, 2018), posicionando el conocimiento vivencial que adquieren los estudiantes en los bordes de los sistemas de pensamiento, para posibilitar posicionamientos alternos al hegemónico (Escobar, 2003).

Aprendí, a través de la experiencia con el caso de mi microempresa, que lo que vemos en la teoría del curso no necesariamente se cumple en realidad. Pude observar que la realidad a veces es diferente o supera la teoría. (Estudiante, 2018-1)

La posibilidad de encontrar que la teoría mainstream resulta insuficiente para contener las realidades que se identifican en el trabajo conjunto entre empresarios y estudiantes, y la dificultad que en principio 
representa para los estudiantes el lograr salir del esquema de conceptos estándar para integrar, proponer y argumentar otros modos de entender interpretativamente a las organizaciones con las que se trabaja, nos hace conscientes de los modos en que seguimos gobernados y colonizados, de formas mucho más sutiles y potentes, por conocimientos, técnicas y prácticas que se sustentan en jerarquías epistémicas producidas en el primer mundo (Lander, 2000 y resguardadas como verdades absolutas desde paradigmas y campos de conocimiento legitimados (Castro-Gómez \& Grosfoguel, 2007.

Esta experiencia confirma que nuestro contexto latinoamericano requiere del desarrollo de una psicología acotada al mismo, lo que exige formar psicólogos orientados al cuestionamiento reflexivo para un ejercicio profesional más consciente, con una mirada decolonial, que si bien conoce y domina las teorías globales, las puede interpelar y reinventar desde las demandas particulares del contexto local, reconociendo que los intereses rectores del conocimiento se forman en el medio del trabajo, del lenguaje y de los sistemas de dominación (Habermas, 1990, en este caso desde la hegemonía académica sobre los saberes del management y del campo organizacional.

Con este proyecto, los estudiantes han experimentado vivencias que contrastan la teoría de clase con lo que se hace, piensa y siente en el entorno laboral real. El trabajo de los estudiantes en cada semestre ha sido arduo y comprometido, encarando algunas dificultades operativas que también potencian el aprendizaje situado que persigue el curso. Este compromiso lo reconocen los empresarios en testimonios brindados al finalizar el proceso cada semestre:

Me gustó la intervención. Si bien son estudiantes, me ayudaron a identificar algunos puntos que son bastante especiales en
mi organización y que debo tener más en cuenta. Yo estoy casi todo el tiempo ocupado con las rutinas diarias. Por eso mismo,
también estoy agradecido por el tiempo que le dedicaron, por ponerse a observar cada uno de los detalles, desde el local hasta
los cocineros. Creo que darse ese tiempo puede generar cosas valiosas y ellas me lo han demostrado con las devoluciones
acerca de lo que vieron. (Restaurante, 2016-1)
Me gustó el Proyecto y espero haber podido ayudarlos con su investigación. Fue algo nuevo. Nunca había recibido visitas
de este tipo, pero creo que pudimos ayudarles y ellos nos ayudaron también a nosotros. Aprendí mucho con las devoluciones
que me hacían sobre lo que pasaba en la lavandería y ellos aprendieron también de lo que hacíamos. No somos una empresa
normal ya que llevamos bastante tiempo trabajando juntos, así que siempre es bueno ver los casos particulares. (Lavandería,
$2017-1$ )
El trabajo de las estudiantes y su retroalimentación significó una ayuda especial pues me ayudaron a identificar estos
conceptos y procesos dentro de una empresa que nosotros, gracias a la experiencia, sabíamos que funcionaban, pero los
habíamos podido ver de manera clara. Fue un proceso de aprendizaje bastante valioso y esperamos haber podido aportar
también al aprendizaje de ellas. (Empresa de Lavado de autos, 2016-2)

Esta propuesta también permite reflexionar sobre mecanismos para buscar el fortalecimiento de vínculos entre la academia y la realidad empresarial más inmediata, desde el ejercicio formativo de psicólogos en las organizaciones locales, reconociendo el lugar de enunciación que cada uno de los actores ejerce y legitimando el valor de un conocimiento que no debería considerarse marginal, periférico o menos relevante que el que se produce en contextos académicos de Norteamérica o Europa (Castro-Gómez \& Grosfoguel, 2007; Quijano, 2000b). Además, se reconoce a los microempresarios como agentes sociales con un conocimiento práctico enriquecido y situado que no debe ser subalterno porque al abstraerse de la supuesta neutralidad positivista o 'punto cero' del conocimiento nórdico, adquiere el potencial para favorecer el cambio y la inclusión social, aunque sus prácticas no estén visibilizadas en los temas de investigación más convencionales del campo de la psicología organizacional.

Se concibe entonces el trabajo entre academia y microempresas como 'heterárquico', que -como explican Castro-Gómez y Grosfoguel (2007) - consistiría en concebir estructuras complejas de relación en las que todos los niveles ejercen algún grado de influencia mutua sobre los otros, por lo que no existe un nivel básico que gobierna sobre los demás. No es la academia desde una posición de poder hablando sobre microempresas, sino microempresarios y estudiantes hablando sobre sí mismos e interpelando a la academia, por lo que las relaciones de mutua afectación se dan de acuerdo a los contextos que establecen otras rutas para construir y 
deconstruir conocimientos, rebatiendo la idea de una lógica determinista, donde el dominio autónomo de unos dispositivos sobre otros desaparece y se generan nuevas 'zonas de contacto'.

Se trata de plantear una relación de saberes ecológica (De Sousa, 2006), de afuera a adentro de la universidad donde las voces "populares" o no académicas tengan el poder suficiente para generar una comprensión situada de las problemáticas locales (Velázquez, Rivera \& Custodio, 2018). La Universidad, con sus especialidades, dejaría de ser un refugio privilegiado para las epistemes, que administran y controlan los conocimientos y metodologías consagrados (Castro-Gómez, 2007) para abrirse al diálogo transdiciplinario y, saliendo a la realidad práctica en que se viven las dinámicas organizacionales locales, configurar también un saber intercontextual, orgánico, transcultural, donde confluyen tipos de saberes y donde diferentes formas culturales de conocimiento pueden convivir en el mismo espacio universitario y fuera de éste porque "decolonizar el conocimiento significa descender del punto cero y hacer evidente el lugar desde el cual se produce ese conocimiento", y precisamente a eso busca llegar el trabajo del curso de que trata este documento.

En síntesis, es relevante reconocer el nivel de impactos positivos del proyecto en varias dimensiones: la primera, a nivel académico, atañe al posicionamiento crítico, reflexivo e interpelador para nuestros estudiantes sobre el rol de la psicología en el mundo del trabajo; aportando, además, una experiencia formativa sistematizada que puede ser transferible a otros contextos y disciplinas en donde se busque propiciar un ejercicio crítico sobre el rol profesional en el contacto directo con la realidad. Nuestros estudiantes manifiestan el deseo de trabajar en ámbitos similares a la propuesta del curso, resaltando el aporte social que puede lograr la psicología en las organizaciones. Desde esta experiencia se han desarrollado tres tesis de pregrado que evidencian el ejercicio del pensamiento crítico tras participar en el proyecto. Egresados que pasaron por el programa reconocen el valor único que el mismo les ha dado en su actual ejercicio profesional. Así que la academia se ve beneficiada con un programa de este tipo porque los aprendizajes son más sólidos, reflexivos y socialmente situados.

La segunda dimensión: la organizacional. Para las empresas participantes se brinda un diagnóstico que de acuerdo con el reporte de los participantes, es de plena utilidad en la toma de decisiones organizacionales, sobretodo porque es construido conjuntamente privilegiando un proceso acotado a las singularidades y necesidades de cada organización; además, a partir de los hallazgos del proyecto se ha encontrado la oportunidad de generar un programa de formación en herramientas de gestión organizacional para los empresarios participantes, que va a repercutir positivamente en sus empresas y empleados.

Finalmente, en la dimensión social, y en relación con el impacto organizacional -dado que en el Perú las microempresas son intensivas en mano de obra-, el favorecer su estudio y su participación en la generación de conocimiento sobre sí mismas, conlleva un aporte en la mejora de sus procesos, sostenibilidad y toma de consciencia de sus responsabilidad social como generadoras de crecimiento y bienestar para sus trabajadores y la sociedad. Así que al divulgar experiencias formativas prácticas como esto, se contribuye a la construcción de rutas para producir conocimientos en pro de una sociedad mejor desde el ámbito del trabajo. Se considera valioso poder compartir esta experiencia con miras a convocar otros saberes que se sumen al encuentro de voces motivadas a comprender el CO situado en el ámbito microempresarial, reafirmando que el contexto sociocultural y económico peruano contempla retos para el desarrollo de una psicología del trabajo pertinente, crítica y socialmente relevante.

\section{Lecciones prácticas aprendidas}

Las lecciones del manejo pedagógico son promover en los estudiantes una motivación identificada desde el inicio del curso, por medio del planteamiento de objetivos y el diseño de una metodología. Brindar a los estudiantes sólidas bases epistemológicas de orientación crítica junto con rigurosas herramientas metodológicas potencializa su capacidad de responsabilidad, agencia y desarrollo de conocimiento activo. 
Para ello, el seguimiento exhaustivo y continuo de sus procesos de campo se vuelve fundamental y la contextualización de la teoría a la práctica son claves en el logro de esta meta. Sin el trabajo del campo el curso no tendría la misma potencia y capacidad transgresora y critica que a lo largo de su ejecución ha desarrollado.

En relación con aprendizajes para la formación e investigación universitaria el proyecto es un excelente semillero para promover la investigación cualitativa, profunda, responsable y crítica en el ámbito de las organizaciones. Un aspecto poco trabajado en el contexto contemporáneo local. Así que, respecto a este punto, la experiencia lo que alecciona es a llevar el registro puntual y completo de toda la información recogida y procesada por los estudiantes durante sus trabajos de campo, con miras a que ello pueda ser usado en la producción de conocimiento académico de alto nivel que informe y explique sobre las condiciones del comportamiento organizacional en las microempresas locales. Por otra parte, varios estudiantes desarrollaron un interés genuino por dar continuidad a la visión del mismo por medio de sus trabajos de grado u otros proyectos de RSU, lo cual promete una plataforma para dar continuidad a la reflexión que plantea el proyecto.

\section{Referencias}

Alva, E. (2017. La desaparición de las microempresas en el Perú. Una aproximación a los factores que predisponen a su mortalidad. Caso del Cercado de Lima. Economía y Desarrollo, 158(2, 76-90.

Amat y León, C. (2012 El Perú nuestro de cada día. Nueve ensayos para discutir y decidir. Lima: Universidad del Pacífico.

Ameconi, O. (2004. Microempresas en acción. Buenos Aires: Macchi.

Amorós, J., \& Bosma, N. (2013. Global Entrepreneurship Monitor 2013 Global Report. Disponible en línea http://w ww.gemconsortium.org/docs/download/3106

Arbulú, J. \& Otoya, J. (2006. La PYME en el Perú. Revista de Egresados. ESAN, Perú. Recuperado de: http://cendo c.esan.edu.pe/fulltext/e-journals/PAD/7/arbulu.pdf

Arellano, R. (2010. Al medio hay sitio: el crecimiento social según los estilos de vida. Lima: Planeta.

Arteaga, A. (2017. Caracterización del financiamiento De las micro y pequeñas empresas del Sector comercio del Perú: caso Empresa grupo Carlos SAC-Casma 2016. Tesis de pregrado. ULAECH, Lima.

Avolio, B., Mesones, A. \& Roca, E. (2011. Factores que limitan el crecimiento de las Micro y Pequeñas Empresas en el Perú (MYPES. Strategia, 22, 70-80.

Bruce, J. (2007. Nos habiamos choleado tanto. Psicoanálisis y racismo. Lima: Fondo Editorial de la Universidad San Martin de Porres.

Campaña, A. (2013. ¿Qué sucede con las microempresas en el Perú? Recuperado en agosto de 2018 de http:// www.saberescompartidos.pe

Carvajal, L.., Ulloa, I., \& Morales, M. (2007 La utilización de pruebas psicométricas: ¿un absurdo en un EST? Universitas Psychologica, 6(1,79-87.

Castro-Gómez, S. \& Grosfoguel, R. (2007. Prólogo. Giro decolonial, teoría crítica y pensamiento heterárquico. En S. Castro-Gómez \& R. Grosfoguel (eds.)El giro decolonial. Reflexiones para una diversidad epistémica más allá del capitalismo global (pp. 9-23. Bogotá: Iesco-Pensar-Siglo del Hombre Editores.

Castro-Gómez, S. (2007. Decolonizar la universidad. La hybris del punto cero y el diálogo de saberes. S. Castro-Gómez \& R. Grosfoguel (eds.), Elgiro decolonial. Reflexionespara una diversidad epistémica más allá del capitalismo global (pp. 79-91). Bogotá: Iesco-Pensar-Siglo del Hombre Editores.

De Sousa, B. (2006). La universidad en el siglo XXI. Para una reforma democrática y emancipadora de la universidad. Umbrales: Revista del Postgrado en Ciencias del Desarrollo, 15, 13-70.

Elizade, R., Mollo, M., \& Flores, J. (2010). Psicología organizacional: aplicación en la vida profesional. Revista de Investigación en Psicología, 13(2), 243-248.

Escobar, A. (2003). Mundos y conocimientos de otro modo: el programa de investigación de modernidad/colonialidad Latinoamericano. Tabula Rasa, 1(1), 51-86. 
Espinosa, A., \& Cueto, R. (2014). Estereotipos raciales, racismo y discriminación en América Latina. En E. Zubieta, J. Valencia, \& G. Delfino (Coords.), Psicología social y política: procesos teóricos y estudios aplicados (pp. 431-442). Buenos Aires: EUDEBA.

Espinosa, A., Calderón, A., Burga, G., \& Güímac, J. (2007). Estereotipos, prejuicios y exclusión social en un país multiétnico: el caso peruano. Revista de Psicología, 25(2), 295-338. Recuperado de: http://revistas.pucp.edu.pe /index.php/psicologia/article/view/1415

Geertz, C. (1996). La interpretación de las culturas. Barcelona. Gedisa.

Guber, R. (2001). La etnografía: método, campo y reflexividad. Bogotá: Norma.

Guber, R. (2004). El salvaje metropolitano: reconstrucción del conocimiento social en el trabajo de campo. Buenos Aires: Paidós.

Gutiérrez, E. (2007). Cultura, organizaciones e intervención. Universitas Psychologica, 6(1), 115-129.

Habermas, J. (1990). Conocimiento e interés. Buenos Aires: Taurus.

Hernani, M., \& Hamann, A. (2013). Percepción sobre el desarrollo sostenible de las Mype en el Perú. Revista de Administração de Empresas, 53(3), 290-302.

Highhouse, S., \& Schmitt, N. (2013). Chapter 1: A snapshot in time: Industrial Organizational Psychology. En N. Schmitt, \& S. Highhouse (eds.), Handbook of Psychology (pp. 643-676). New Jersey: John Wiley \& Sons, Inc.

Instituto Nacional de Estadística e Informática - INEI. (2018). Evolución de la pobreza Monetaria 2007-2017. Recuperado el 20 de julio de 2018 de: https://www.inei.gob.pe/media/cifras_de_pobreza/informe_tecnico_p obreza_monetaria_2007-2017.pdf.

Kogan, L., Fuchs, R., \& Lay, P. (2013). No... pero si: Discriminación en empresas de Lima Metropolitana. Lima: Universidad del Pacífico.

Lander, E. (2000). ¿Conocimiento para qué? ¿Conocimiento para quién? Reflexiones sobre la universidad y la geopolítica de los saberes hegemónicos. Estudios Latinoamericanos, 1(12), 25-46.

León, F. (2013). Las psicologías del área social-organizacional en el Perú: 2003-2012. Revista de Psicología, 31(2), $177-226$.

Mandiola, M. (2013). Management education in Chile: From politics of pragmatism to (im)possibilities of resistance. Universitas Psychologica, 12(4), 1085-1098. doi: 10.11144/Javeriana.UPSY12-4.meic

Mandiola, M. (2018). La mirada decolonial en el management. Revista Gestión y Tendencias, 3(1), 6-10. DOI:10.11565/gesten.v3i1.53

Matute, G., Bohorquez, K., Carbajal, L., Díaz, C., Espinoza, A., \& Jiménez, C. (2008). Segmentación psicográfica de la pequeña y microempresa. ESAN Ediciones.

Miller, P. \& Rose, N. (2009). Governing the present. Administering economic, social and personal life. Cambridge: Polity Press.

Portocarrero, F., Tarazona, B., \& Camacho, L. (2006). Situación de la responsabilidad social empresarial en la micro, pequeña y mediana empresa en el Perú. Lima: Centro de Investigación de la Universidad del Pacífico.

Pulido-Martínez, H. (2004). En búsqueda de una psicología crítica en los ámbitos laborales. Universitas Psychologica, $3(2), 213-222$.

Pulido-Martínez, H., \& Sato, L. (2013). Y entonces ¿esto de la crítica qué es? De las relaciones entre la psicología y el mundo del trabajo. Universitas Psychologica, 12(4). DOI:10.11144/Javeriana.UPSY12-4.rpmt

Pulido-Martínez, H., \& Jaraba, B. (2017). Entre psicologías, prácticas y descentramientos: O de constitución de la subjetividad de los psicólogos. Psicoperspectivas, 16(3), 99-109. http://dx.doi.org/10.5027/psicoperspectivas-vo 116-issue3-fulltext-1078

Quijano, A. (2000a). Colonialidad del poder y clasificación social. Journal of World-System Research, 1(2), 342-386.

Quijano, A. (2000b). Colonialidad del poder, eurocentrismo y América Latina. En E. Lander (ed.), La Colonialidad del saber: Eurocentrismo y Ciencias Sociales. Perspectivas Latinoamericanas (pp. 201-245). Caracas: Clasco.

Ravelo, J. (2012). Las mypes en el Perú. Strategia, 26, 45-47. 
Robbins, S., \& Judge, T. (2013). Comportamiento organizacional. México DF: Pearson Educación.

Stecher, A. (2013). Un modelo crítico-interpretativo para el estudio de las identidades laborales. Contribuciones a la investigación psicosocial sobre trabajo y subjetividad en América Latina. Universitas Psychologica, 12(4). doi: 10.11144/Javeriana.UPSY12-4.mcie

Tello, Y. (2014). Importancia de la micro, pequeñas y medianas empresas en el desarrollo del país. Lex, 12(14), 199-218. DOI: http://dx.doi.org/10.21503/lex.v12i14.623

Velázquez, T., Rivera-Holguín, M., \& Custodio, E. (2017). Aportes y reflexiones de la formación en psicología comunitaria PUCP en tres regiones del Perú. Revista de Psicología, 35(1), 193-224. https://doi.org/10.18800/ psico.201701.007

Veronese, M., \& Guareschi, P. (2005). Possibilidades solidárias e emancipatórias do trabalho: campo fértil para a prática da Psicologia Social Crítica. Psicologia \& Sociedade, 17(2), 58-69.

Zamora, S. (2017). Caracterización del financiamiento de las micro y pequeñas empresas del sector comercio del Perú: caso empresa "Grupo Culmen SAC", Chimbote, 2016.

\section{Anexo 1.}

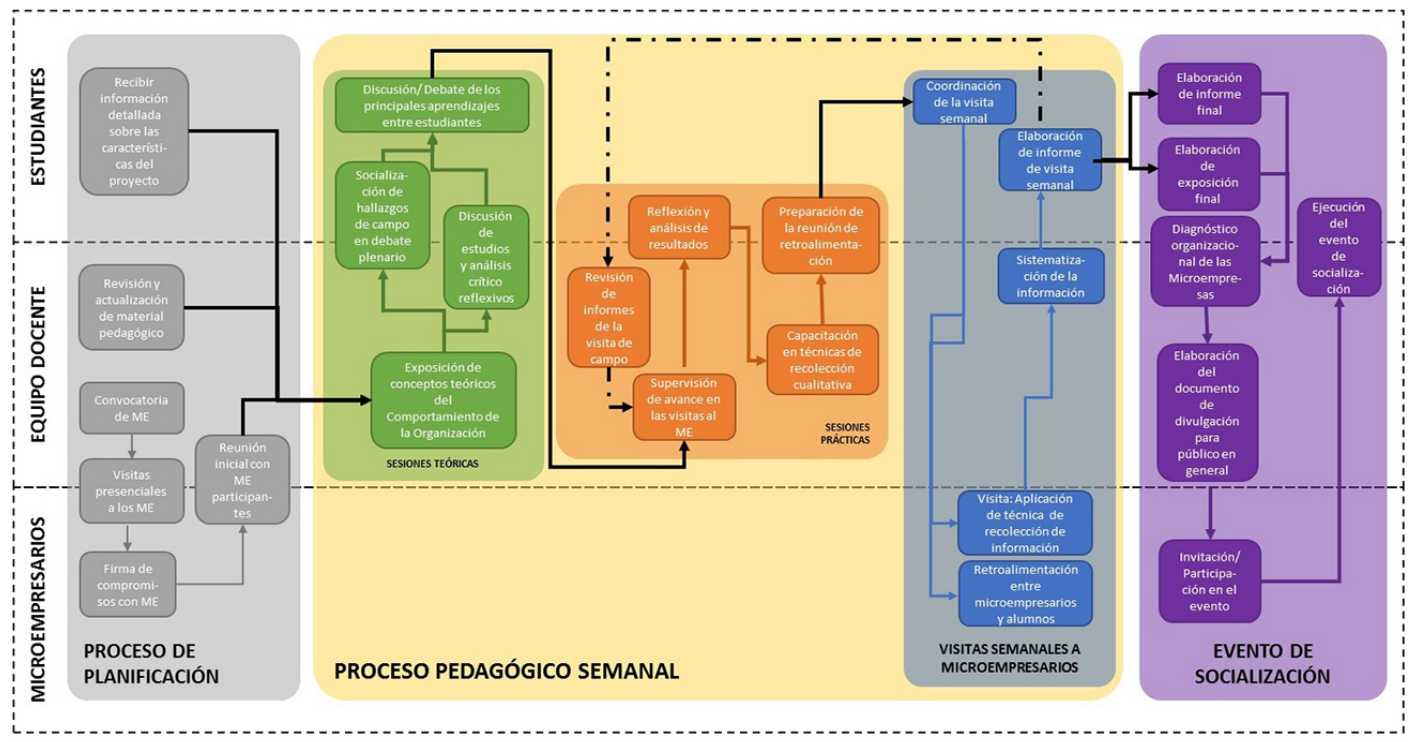

Flujograma del procedimiento pedagógico

Fuente: Elaboración propia

\section{Notas}

* Artículo de investigación científica.

\section{Licencia Creative Commons CC BY 4.0}

Sugerencia de citación: Vera Ruiz, A., Llanco G., C. A. \& Guevara R., L. A. (2019). Desafiando la formación de psicólogos organizacionales desde una perspectiva de aprendizajes situados en el ámbito micro-empresarial peruano. Cuadernos de Administración, 32(58). https://doi.org/10.11144/Javeriana.cao32-58.dfpo 